\title{
Las relaciones interespecíficas de las universidades con sus grupos de interés: metáfora biológica
}

Ana M. Barrera-Rodríguez ${ }^{1,2 *}$, Jaime A. Vieira-Salazar ${ }^{2}$ y Edison J. Duque-Oliva ${ }^{3,4}$

(1) Facultad de Ciencias Económicas, Administrativas y Contables, Universidad Libre, Pereira, Colombia. (correo-e: anam.barrerar@unilibre.edu.co)

(2) Facultad de Administración, Universidad Nacional de Colombia, Manizales, Colombia.

(correo-e: javieiras@unal.edu.co)

(3) Facultad de Ciencias Económicas, Universidad Nacional de Colombia, Bogotá, Colombia.

(correo-e: ejduqueo@unal.edu.co)

(4) ESAI Business School, Universidad Espíritu Santo, Samborondón, Ecuador.

* Autor a quien debe ser dirigida la correspondencia.

Recibido Feb. 10, 2020; Aceptado Abr. 13, 2020; Versión final May. 18, 2020, Publicado Ago. 2020

\section{Resumen}

El presente artículo tiene como objetivo identificar las diferentes relaciones interespecíficas a las que se enfrentan las universidades con sus grupos de interés, a través del uso de la metodología del desarrollo de metáforas biológicas. Las universidades conscientes de su misión reconocen la importancia de las relaciones con sus grupos de interés porque de ellas depende en gran medida su prestigio y reconocimiento. Se encontró que estas relaciones pueden asociarse con las interespecíficas que se dan a nivel biológico. El mutualismo, que puede llamarse "gana-gana", se observa en las relaciones entre la universidad y los estudiantes, los profesores, los funcionarios administrativos, y el sector privado y público; y el parasitismo, que puede ser generado desde adentro por varios grupos con interés individual. Se concluye que los modelos metafóricos biológicos son aplicables en la gestión de las relaciones interespecíficas de las universidades con sus grupos de interés.

Palabras clave: metáfora biológica; relaciones interespecíficas; universidad; grupos de interés

\section{Interspecific relationships of universities with their stakeholders: biological metaphor}

\begin{abstract}
The purpose of this article is to identify the different interspecific relationships that universities face with their stakeholders through the use of the methodology of development of biological metaphors. Universities recognize the importance of their relationships with their interest groups because their prestige and recognition depend largely on these groups. The results show that these relationships can be associated with the interspecific relationships that occur at the biological level. Mutualism, which can be called "win-win," is observed in the relationships between the university and its students, professors, administrative officials, and the private and public sector. Parasitism can be generated from within by various groups with individual interest. In conclusion, biological metaphoric models can be applied to the management of interspecific relationships by universities and their interest groups.
\end{abstract}




\section{INTRODUCCIÓN}

El incremento de la instituciones de educación superior, la normatividad vigente en materia de calidad, la globalización de la economía (Cutovoi, 2018) y la decisión voluntaria de las universidades por la obtención de acreditación de alta calidad, les hace imperativo conocer sus grupos de interés (Duque-Oliva, 2009), las interacciones y sus relaciones, bien sean beneficiosas, inocuas o perjudiciales y de esta manera responder a sus expectativas considerando "aspectos éticos que les permitan cumplir sus obligaciones, sin poner en riesgo los intereses de los públicos que afectan o son afectados por ellas" (Osorio et al., 2018). Siebold (2020), muestra cómo organizaciones con un propósito social integrado pueden innovar sus modelos de negocio mediante el estudio de los grupos de interés como puntos de referencia para teorizar la innovación del modelo de negocio.

La competencia es, sin lugar a dudas, una interacción que está a la orden del día y que lleva a la definición de estrategias que le garanticen a la universidad mantener su prestigio y su supervivencia. Además, así como en las relaciones entre seres vivos se han definido una serie de interrelaciones de mutualismo, competencia, parasitismo, etc., la aplicación de la metáfora biológica que concibe a las organizaciones como seres vivos (Vujnovic y Kruckeberg, 2005) permite la identificación de soluciones creativas para problemas complejos de las empresas (Hernández y Domínguez, 2008), extrapolando el concepto biológico de las relaciones interespecíficas a la de las relaciones entre las organizaciones, por ejemplo, universidades y sus grupos de interés. De esta forma, relaciones tales como el mutualismo, se puede obtener beneficio mutuo, el comensalismo, si bien no se gana, tampoco se pierde, y el parasitismo, uno gana a costa del otro que, indudablemente, se ve afectado y la depredación, interacción más nociva que puede llevar a la muerte de la institución, se ven presentes en el contexto universitario.

Los grupos de interés pueden ser personas, grupos, barrios, organizaciones, instituciones, sociedades e incluso el entorno natural (Mitchell et al., 1997). Freeman et al. (2004) afirman que "cualquiera que sea el objetivo final de la corporación u otra forma de actividad empresarial, los gerentes y los empresarios deben tener en cuenta los intereses legítimos de los grupos e individuos que pueden afectar" y velar porque éstos se cumplan (Chiva-Bartoll et al., 2019). La teoría de los grupos de interés nace en los años ochenta (dos Santos et al, 2019) y los define como "cualquier grupo o individuo que puede afectar o ser afectado por el logro de los objetivos de la empresa" (Freeman, 2010) y en la que se como una metodología de gestión empresarial donde las organizaciones empiezan a concebirse como entes plurales que son influenciados no sólo por sus accionistas o dueños, sino por sus empleados, clientes, proveedores, competidores y la comunidad donde tiene presencia (Plichta, 2019). Por su parte, Clarkson (1995) establece que los grupos de interés son "personas o grupos que tienen, o reclaman, propiedad, derechos, o intereses de una empresa.

Dichos derechos reclamados son el resultado de transacciones con la empresa, y pueden ser legales o morales, individuales o colectivas" y los clasifica en grupos según sus intereses, reclamos y derechos, en grupos de interés primarios y secundarios. El grupo de interés primarios los define como los actores con los que la empresa tiene relaciones constantes dependientes, sin los cuales la empresa no existiría. Entre este grupo se encuentran los accionistas, empleados, clientes, proveedores y actores públicos en los que se encuentra el gobierno (Alonso-Almeida et al., 2019). Los grupos de interés secundarios no vitales para la organización los define como los actores que influyen o son influenciados por la empresa pero que no realizan transacciones con ella, cómo por ejemplo, los medios de comunicación. Existen diferentes clasificaciones de los tipos de grupos de interés que poseen las organizaciones y en el caso de las universidades se pueden encontrar ya aplicaciones previas de los mismos (Duque-Oliva, 2009).

Mitchell et al. (1997), propusieron un modelo donde evalúan los grupos de interés de acuerdo a tres atributos, el poder, la legitimidad y la urgencia, con fin de identificar sus expectativas y la forma en la que la empresa se debe relacionar con ellos. A partir de este modelo y de la combinación de sus atributos plantean que existen siete clases de grupos de interés clasificados de acuerdo con la posesión de un atributo, dos atributos o tres. Los grupos de interés que poseen un solo atributo son conocidos como los latentes divididos en grupos de interés dormidos, discrecionales y demandantes. Los dormidos son grupos de interés que poseen poder ante la organización y que por lo tanto deben tratarse con precaución. Los discrecionales poseen legitimidad debido a que cuentan con unas características que les permiten solicitar apoyos financieros a la organización. Los demandantes poseen urgencia cuyo accionar puede perjudicar a la organización a través de protestas. Los grupos de interés con dos atributos se definen como demandantes que pueden ser dominantes, dependientes y peligrosos. Los dominantes son un tipo de grupo de interés que es clave e importante para la organización debido a que poseen poder y legitimidad sobre la organización. Los dependientes cuentan con legitimidad y urgencia por lo que para las organizaciones son unos grupos de interés que no tienen la posibilidad de hacer valor sus intenciones. Los grupos de interés peligrosos poseen poder y urgencia porque pueden tomar actitudes violentas y de coerción hacia la organización. Por último, los grupos de interés con los tres atributos se conocen como definitivos y son a los que más atención la organización les debe prestar. 
Marulanda et al. (2019) definen que los grupos de interés de las universidades son "personas naturales y jurídicas que poseen influencia en los objetivos, desempeño y resultados organizacionales de la IES, por ende, necesitan clasificarse con base en el nivel de importancia para atender de forma oportuna y eficiente los requerimientos". De acuerdo con la clasificación de Clarkson (1995) los grupos de interés primarios de las universidades se pueden clasificar en estudiantes, profesores, trabajadores administrativos, sindicatos y el gobierno. En el grupo de secundarios están los egresados, medios de comunicación y comunidad en general.

Por su parte Mainardes et al. (2013) afirman que los grupos de interés de las universidades son numerosos y muy variados y que se pueden clasificar como internos o externos, individuales o colectivos, académicos 0 no académicos. Vallaeys et al. (2009) definen los grupos de interés de las universidades como los "públicos interesados primordialmente en el buen desempeño de la universidad" y presentan en total once grupos de interés directos que son el personal no docente, personal docente-investigador, autoridades, estudiantes, proveedores, egresados, empleadores, competidores, comunidades locales, organizaciones socias y el estado. Y como grupos de interés indirectos a "los padres de los estudiantes, los familiares, de los empleados, los colegios profesionales, los sectores del Estado que se vinculan con la universidad, o incluso las generaciones futuras" (Vallaeys et al., 2009). A partir de estos grupos proponen un modelo de clasificación teniendo en cuenta los impactos organizacionales, educativos, cognitivos y sociales que generan las universidades en autoridades y personal docente y no docente, proveedores, estudiantes, docentes e investigadores, sociedad civil, sector privado y público.

Vieira-Salazar (2013) afirma que es indispensable que la organización universitaria adquiera el criterio suficiente para identificar, clasificar y estimar sus grupos de interés, y así dar respuesta eficiente a sus principales expectativas y necesidades, máxime cuando dichas relaciones son de carácter complejo debido a la multiplicidad y heterogeneidad de sus grupos de interés, determinando tres fundamentales de la universidad: las empresas, los grupos de investigación, los estudiantes, los responsables de la gestión académico administrativa y los profesores. De igual forma el autor propone a partir de Mitchell et al. (1997), una clasificación de grupos de interés universitarios de acuerdo con sus atributos de legitimidad, poder y urgencia, en articulación con sus impactos organizacionales, educativos, cognitivos y sociales del modelo de (Vallaeys et al., 2009).

El presente documento pretende exponer la metáfora biológica, como las relaciones interespecíficas de las universidades con sus grupos de interés, para contribuir al conocimiento y comprensión de estas, y a la toma de decisiones en cuanto a estrategias que las lleven al mantenimiento de su prestigio y su supervivencia. Por esta razón, al usar la metáfora biológica, se parte de que las relaciones interespecíficas son las relaciones que tienen los miembros de diferentes especies que pertenecen a una misma comunidad o sistema biológico (Bansah et al., 2018) y que dichas relaciones están unidas por flujos de recursos y de información que pueden ser desde, altamente especializadas, es decir, una sola especie puede proporcionar bienes o servicios que otro requiere, hasta altamente generalizadas y desde, altamente beneficiosas hasta bastante perjudiciales (Mars et al., 2012).

\section{OTROS ANTECEDENTES}

Las relaciones interespecíficas se clasifican en tres grupos: el primero hace referencia a las relaciones que generan perjuicio para las especies involucradas y pueden ser las relaciones de competencia, depredación y parasitismo. El segundo grupo, se da cuando las relaciones entre las dos especies tiene como resultado un beneficio, este es el caso del mutualismo (Mars et al., 2012; Tejani et al., 2016). Por último, se encuentran las relaciones en las que una de las especies se beneficia y la otra no se beneficia, pero tampoco se perjudica (Coccia, 2019; Bansah et al., 2018). La principal relación de este tipo se conoce como comensalismo (Mars et al., 2012; Tejani et al., 2016).

La competencia, según Bansah et al (2018), ocurre cuando dos o más especies deben competir por los mismos recursos, alimentos, hábitat, etc. En este tipo de relación la competencia es más intensa cuando el hábitat de las dos especies es cercano o es el mismo. En ocasiones la relación de competencia que se da entre las dos especies genera que la especie B gane terreno y recursos respecto a la especie A y en otras, que B pierda terreno y A lo gane. En otras situaciones puede darse que una de las especies sea eliminada por la otra.

La depredación es un tipo de relación "breve, entre una especie, el depredador, que persigue, mata y consume a ejemplares de la otra especie, la presa" (Martínez-Fernández y Cordero-del Campillo, 2005). De igual forma, se puede definir como "la interacción entre organismos cuyo resultado puede ser el consumo total o parcial del otro" (Castro-Ríos y Noguera-Hidalgo, 2014) y "ocurre cuando una criatura se alimenta de otra, como los depredadores que comen animales herbívoros, animales herbívoros que comen plantas verdes, parásitos e insectos en el canibalismo" (Zhang et al., 2017). 
El parasitismo viene del griego para que significa cerca, y de sitos que significa alimentos y puede definirse como la "relación que tienen individuos de dos o más especies, en la cual los individuos de una o más de ellas derivan beneficios, perjudicando al otro, pero sin ocasionarle la muerte" (Hilje, 1984). El parasitismo es una forma de relación interespecífica en la que un organismo se beneficia a costa de otro, y eso otro se perjudica hasta el punto de que puede llevarlo a la muerte (Robinson, et al., 2019). El mutualismo en la biología se define como una relación en la que dos organismos se benefician el uno del otro (Coccia, 2019). De igual forma, Robinson, et al. (2019) afirma que en el mutualismo los organismos pueden "coexistir, beneficiarse unos de otros y, en última instancia, incluso dar lugar a un nuevo tipo de organismo". Para Bascompte (2019) el mutualismo es "un tipo de interacción en la que ambos socios se benefician de cada uno". En comensalismo, según lo expresado por Robinson, et al. (2019), es un tipo de relación entre especies diferentes en la que uno de los individuos se beneficia más de la simbiosis. Otra definición establece que el comensalismo es un tipo de "relación entre dos organismos donde un organismo se beneficia del otro sin afectarlo" (Coccia, 2019).

\section{METODOLOGÍA}

La metodología propuesta en este trabajo se fundamenta en el método un cualitativo comparativo (Saunders et al., 2016) que permite identificar similitudes y diferencias entre los casos de estudio (Barrera-Rodríguez et al., 2018) y de naturaleza descriptiva (Saunders et al., 2016) a través del análisis bibliográfico y la aplicación de un modelo metafórico, que es reconocido "como un potente dispositivo de creación de ciencia" (Martínez et al., 2017) en el que se comparan procesos biológicos desarrollados desde el discurso científico (Villaneda, 2015) con diferentes procesos que se originan en las organizaciones y cuyo enfoque permite la construcción de nuevas perspectivas epistemológica y el desarrollo de nuevas formas de abordar y entender la gestión de las organizaciones (Bueno et al., 2006).

La metáfora es "...un recurso literario que consiste en identificar dos términos entre los cuales existe alguna semejanza" (Montoya-Restrepo et al., 2010). Según Martínez et al. (2017) "la metáfora se presenta como constructora de ciencia, porque al tener la ciencia su propio lenguaje, encuentra otras maneras atractivas de presentar sus propias ideas". De igual forma, las metáforas son muy utilizadas cuando se quiere explicar de la forma más sencilla posible algo abstracto o difícil de entender (Clarke et al., 2014; Montoya-Restrepo et al., 2010), por lo que pueden considerarse como "herramientas que se utilizan para dar forma a las realidades" (Bueno et al., 2006). Es así como las "metáforas generan significados emergentes y crean similitudes a medida que se construyen correspondencias entre dominios de orden superior" (Clarke et al., 2014).

Para Lakoff y Johnson (1990) las metáforas hacen parte de la vida cotidiana de las personas cuyo "sistema conceptual ordinario es de naturaleza metafórica". A partir de su publicación denominada Metáforas de la Vida Cotidiana, propusieron que las metáforas podían clasificarse en tres categorías, así: i) Metáforas orientacionales: sistematizan una red global de conceptos en relación con otros. Nacen de nuestra interacción con el mundo físico. Se vinculan, mayormente, a la orientación espacial: arriba/abajo, derecha/izquierda, dentro/fuera, delante/detrás, profundo/superficial, central/periférico; ii) Metáforas ontológicas: categorizan un fenómeno de forma peculiar mediante su consideración como entidad, sustancia, recipiente, individuo, etc. para tornar sus rasgos constitutivos más concretos y tangibles; y iii) Metáforas estructurales: organizan una actividad o noción en términos de otra (Martínez et al., 2017)

Las metáforas biológicas son "herramientas útiles para comprender y predecir las condiciones que dan forma e influyen en los sistemas organizacionales" (Mars et al., 2012). Robayo et al. (2011) afirman que el uso de las metáforas para aplicarlas a conceptos de las ciencias de la gestión tiene sus orígenes en las teorías administrativas, con el aporte de Frederick Taylor a la teoría de la Administración Científica, quien describe a la organización como una máquina. De igual forma, Bateson en 1972 estableció la metáfora del pensamiento sistémico (Robayo et al., 2011).

De acuerdo con Montoya-Restrepo et al. (2010), diferentes autores han aplicado metáforas biológicas a distintos temas de la gestión organizacional. Este es el caso de Beer que en 1977 relacionó la organización con otras ciencias; de Illera que en 1982 planteó la semejanza que tienen las organizaciones con el cuerpo humano; de Lessem que en 1990 toma la estructura de los árboles para explicar la teoría organizacional; y de Morgan, quien es considerado el autor pionero en este tema, que en 1991 plantea las organizaciones como máquinas, organismos, cerebros, culturas, sistemas políticos y cárceles psíquicas. A partir de allí, se han desarrollado diferentes metáforas cuyo objetivo es proporcionar al lector información que simplifique el concepto organizacional y que permita, por tanto, su entendimiento. Una de las metáforas más usadas es la metáfora biológica. Para Montoya-Restrepo et al. (2016), la ventaja principal de la aplicación de metáforas biológicas en las ciencias de la gestión es que permite la comprensión de las relaciones que se dan entre las organizaciones y el ambiente en el que se encuentran y en el que "el lenguaje de las ciencias naturales, especialmente de la biología, tiene un valor considerable para ayudarnos a comprender los fenómenos de las ciencias sociales" (Robinson, et al., 2019). 
Según la revisión sistemática de literatura realizada, para Robayo et al. (2011) la mayor cantidad de estudios en los que se han aplicado las metáforas biológicas se han enfocado en el desarrollo de las ciencias económicas, en los modelos evolucionistas y en la teoría de gestión de las organizaciones, principalmente en: el eje temático de conceptos de integración, con un énfasis hacia la formación de comunidades ecológicas y nichos, donde se evalúan las estructuras organizacionales con un enfoque hacía la organización ecológica, vista desde diversas características, como la inercia, el nicho, la mortalidad, como una forma de organización y estructuración frente al cambio organizacional (Robayo et al., 2011). La metáfora biológica, entonces, adquiere significado en tanto se describan las relaciones que se presentan entre la organización y sus principales grupos de interés.

\section{RESULTADOS Y DISCUSIÓN}

La concepción de las organizaciones, como organismos o ecosistemas biológicos, permite entenderlas como sistemas vivos abiertos que necesitan sobrevivir y crecen en medio ambiente del cual dependen para satisfacer sus variadas necesidades (Tohidian et al., 2019). Tanto los sistemas de las organizaciones 0 biológicos "involucran múltiples actores que interactúan positiva y negativamente como pares y como grupos, y flujos de recursos que pueden formarse o desarrollarse a partir de estas interacciones" (Mars et al., 2012). Para Ganhao (2016) esta concepción abarca tanto las relaciones que surgen con los diferentes actores internos como las relaciones externas que se producen con el entorno y medio ambiente en el que se desenvuelven.

Las universidades son organizaciones sociales con "funciones claramente establecidas y exigidas por parte de la sociedad, en lo que se refiere a producción y difusión del conocimiento, al igual que a la formación de profesionales, dirigentes y líderes de la sociedad" (ASCUN, 2011). Aldeanuava-Fernández (2013) afirma que "el carácter universal de la universidad provoca que la inmensa mayoría de los grupos de interés tengan relación, directa o indirecta, con dicha institución". En las universidades, las relaciones interespecíficas de competencia son parte de la cotidianidad. Garde-Sánchez et al. (2013) afirman que existe un alto nivel de competencia en el sector académico. Sin importar si son universidades privadas o públicas, viven constantemente enfrentándose a situaciones en las que deben competir con otras universidades por estudiantes, por recursos humanos y financieros, y por la obtención de altos niveles de reputación. En el mundo Ministerios de Ciencia, Tecnología e Innovación y otros organismos co-financiadores publican convocatorias para universidades y centros de investigación con el objetivo de financiar proyectos, otorgar becas y mejorar las capacidades científicas en diferentes áreas del conocimiento, lo que genera primero, una carrera desenfrenada en la construcción de proyectos de investigación y desarrollo; segundo, el establecimiento de alianzas y tercero, un intenso lobby político para tratar de ganar y/o sacar de la arena a los competidores menos fuertes. Dicha competencia no siempre termina en que gana el mejor sino el que tenga mejores alianzas o relaciones políticas.

De igual forma, la globalización ha generado que la competencia trascienda las fronteras y que no sólo se presente en mercados cercanos, sino que se de con universidades nacionales e internacionales, en educación presencial, semi presencial y virtual, lo que ha producido que las universidades adopten estrategias de expansión y de diferenciación. Actualmente se publican a través de todos los medios de comunicación ofertas de universidades extranjeras de programas que van desde pregrado hasta doctorado $100 \%$ virtuales o con muy escasa presencialidad que compiten fuertemente con los programas de las universidades locales y tradicionales.

En organizaciones como las universidades, la relación de depredación, según Castro-Ríos et al. (2014), se puede definir como "actos de atacar o saquear, pillaje, robo con violencia, devastación, malversación o exacción injusta por abuso de autoridad o de confianza". En este tipo de depredación, la universidad es la presa y los estudiantes, profesores y los administrativos, son los depredadores. Otra concepción más tradicionalista afirma que la relación de depredación surge entre diferentes universidades, donde las más grandes, antiguas, prestigiosas y con mayores recursos, a través de estrategias de mercadeo como la fijación de precios, la disponibilidad de programas, entre otros, consumen a universidades pequeñas que no tienen la posibilidad de competir (Taylor, et al., 2013). Un ejemplo ilustrativo de esta relación es la utilización que hizo una universidad prestigiosa en conjunto con una emisora de alta circulación nacional de una estrategia de mercado tendiente a despertar la generosidad de las personas y empresas para donar matriculas de estudiantes y así, sin mayor esfuerzo conseguir un número apreciable de nuevos estudiantes en detrimento de otras universidades que no cuentan con el mismo nivel de reconocimiento.

En la actualidad han surgido nuevos depredadores, tales como las pequeñas universidades con altos niveles de innovación y capacidad de adaptación que están persiguiendo a sus antiguos depredadores, las universidades tradicionales; este es el caso de universidades relativamente nuevas que están implementando 
formas de educación a distancia, virtual y semi presencial con unos valores de matrícula ostensiblemente más bajos con las que están arrebatando estudiantes a las universidades tradicionales (Taylor, et al., 2013). Otro tipo de depredación se da cuando el gobierno es el depredador y la presa es la universidad, específicamente en el caso de las universidades públicas cuando se establecen procesos de privatización que son "afectadas por las políticas de corte neoliberal, por las restricciones presupuestarias, por el ajuste fiscal y la transformación del contrato social entre el Estado y la sociedad civil" (Mollis, 2003). También existe la depredación tecnológica que es la apropiación o uso sin permiso de una nueva tecnología por parte de un "tecnopredador" en detrimento de su creador, este tipo de relaciones ocurre entre una universidad y una empresa y una universidad e investigadores o inventores (Mesly, 2019). Un ejemplo de depredación tecnológica sucede cuando un investigador perteneciente a una universidad desarrolla un prototipo cuyos derechos morales son de él y los patrimoniales de la universidad. Como la universidad ve el potencial económico del prototipo decide desconocer al investigador y su autoría, argumentando que si trabaja con la universidad nunca será dueño de ningún invento o desarrollo.

La relación de parasitismo puede evidenciarse frente a la actuación de algunos grupos de interés de las universidades, cuyo accionar eventual se hace de manera violenta y coercitiva con paros ilegales, sabotajes, terrorismo, entre otros, en ocasiones mediando excesos en exigencias en beneficio propio, y que está determinado por la urgencia en sus solicitudes o pretensiones sin contar con la necesaria legitimidad (VieiraSalazar, 2013), por cuanto este tipo de acciones impiden u obstaculizan el quehacer universitario y les generan condiciones de sujeción hacia ellos en detrimento administrativo y por supuesto económico, hecho que puede incluso poner en peligro su subsistencia.

El mutualismo se da entre la universidad y los estudiantes, los profesores, los funcionarios administrativos, y el sector privado y público debido a que cada uno de ellos se beneficia de la relación. Los estudiantes y la universidad se benefician mutuamente de su relación: los estudiantes porque adquieren conocimientos y la posibilidad, una vez que se gradúen, de ejercer una profesión de forma legal, y las universidades porque a través del pago de las matrículas, y otros servicios, generan ingresos que conllevan posibilidades de crecimiento vía reinversión. Otro ejemplo de mutualismo se presenta en las empresas que desarrollan su actividad dentro de la universidad, tal como cafeterías, fotocopiadoras y otros servicios conexos adicionales que se ofrecen en el campus. En estos casos, la universidad cobra un alquiler por el uso de los espacios y, en ocasiones, algún porcentaje de las ganancias. A cambio de ello, estas empresas se benefician por tener la población objeto cautiva, hecho que les facilita la venta de sus productos.

De acuerdo con Caja-Vivcancos y García-Gómez (2016) las universidades también tienen relaciones mutualistas con el sector privado, el público y los estudiantes; éstas relaciones se dan a partir de la función de extensión universitaria, entendida, grosso modo, en la transferencia del conocimiento pragmático generado en la universidad al servicio de la sociedad en la solución, o contribución a la solución, de problemáticas sociales, ambientales y económicas, como es el caso de la formación continuada, la asesoría empresarial, la consultoría, la interventoría, entre otras. Ejemplo cotidiano se da cuando las universidades solicitan plazas para prácticas para sus estudiantes; en estas relaciones los tres actores ganan. El sector público y privado se beneficia gracias al trabajo desarrollado por los estudiantes; ellos, por su parte se benefician con la posibilidad de aplicar sus conocimientos y con la adquisición de experiencia; y las universidades, a su vez, con la coformación de sus discentes, que solo el pragmatismo aplicativo puede lograr (Robinson, et al., 2019). Igualmente, se dan relaciones de mutualismo en la relación universidad, empresa, estado, cuando a través de alianzas, todas obtienen beneficio de su interactuación.

El comensalismo en las universidades se da tanto con grupos de interés primarios como secundarios (Clarkson, 1995), los cuales pueden beneficiarse de la relación mutua, recibiendo los efectos de la actividad universitaria. Tal es el caso de las asociaciones de egresados y otras semejantes que se alojan dentro de la institucionalidad universitaria recibiendo sus beneficios y aportando en términos generales, quizás muy poco, al desarrollo académico de las universidades. Otra forma de comensalismo se da en las unidades de emprendimiento de las universidades, a las que acude la comunidad universitaria con sus ideas de empresarismo para recibir la orientación y apoyo en la creación de empresas, en este caso la universidad no se perjudica y quienes se benefician son los que acuden a la unidad.

\section{DISCUSION FINAL}

Las relaciones que establecen las universidades con sus diferentes grupos de interés aparecen complejas y difíciles de comprender. En efecto, un mismo grupo de interés, en un momento dado, puede ser docente y estudiante, puede ser benefactor o deudor; un paso dentro del aula se es docente, un paso afuera se es colega. Una empresa bien puede ser receptora de la gestión del conocimiento o contratista. Es así, como dependiendo del contexto un grupo de interés puede actuar como un comensal, pero durante una situación específica puede convertirse en un mutualista a corto plazo, pero si la situación anterior continúa, se convierte 
en un parásito y posteriormente puede acabar con la universidad al actuar como un depredador. A las relaciones anteriores en biología se les conocen como el continuo simbiótico que pueden variar desde el mutualismo al comensalismo y posteriormente al parasitismo (Aly et al., 2011; Saikkonen et al., 1998). Se quiere significar que las relaciones de las universidades con sus grupos de interés deben ser analizadas en contextos, basadas en roles específicos y en modelos simples de comprensión.

El desarrollo de las universidades, por ejemplo, ha estado ligado a intereses de grupos de interés específicos, como la industria, y en ocasiones en contravía con uno de los fines clásicos de la universidad: la equidad social (Duque-Oliva, 2009). No obstante, para Misas (2004), la universidad no debe ser vista como una ONG, ni relevar al Estado; su responsabilidad no puede ser concebida como un organismo de ayuda social. Lo que sí debe hacer, es contribuir, mediante la creación y divulgación de conocimiento, en la solución de problemas sociales mientras estén en su función social. Esta, entonces, es particular y diferente a la empresa productiva; por ende, sus relaciones con sus principales grupos de interés.

Es posible afirmar que las universidades, como organizaciones, permanentemente sostienen relaciones con sus grupos de interés en las que obtienen beneficios mutuos (comensalismo y mutualismo) y en otros casos, pueden verse perjudicadas (depredación y parasitismo). Al utilizar las relaciones interespecíficas de las especies como metáfora biológica se posibilita la comprensión de una manera simple y cotidiana de las relaciones de las universidades con sus grupos de interés y la identificación de la forma en las que las universidades pueden gestionarlas, estableciendo estrategias para minimizar las afectaciones y generar ventajas que les permita permanecer en el mercado (Huerta-Riveros, et al., 2020).

\section{CONCLUSIONES}

De acuerdo con el trabajo presentado y los resultados obtenidos, se pueden plantear las siguientes conclusiones principales. Primero, los modelos metafóricos biológicos son aplicables en la gestión de las relaciones interespecíficas de las universidades con sus grupos de interés. Segundo, el uso de los modelos metafóricos biológicos permite a las universidades que la gestión se desarrolle de una manera más dinámica y apropiada porque parte de la identificación clara de las posibles relaciones simbióticas que se establecen con los grupos de interés. Tercero, así como las universidades tienen unos intereses derivados de su misión, los grupos de interés tienen también intereses propios y el uso de los modelos metafóricos biológicos expuestos en el artículo posibilita el conocimiento de las diferentes tipos de relaciones interespecíficas para crear una sinergia en la que se cambien las relaciones que generan perjuicio a unas que generen beneficios para todas las partes.

\section{REFERENCIAS}

Aldeanuava-Fernández, I. Los grupos de interés en el ámbito de la responsabilidad social universitaria: un enfoque teórico. Anuario Jurídico y Económico Escurialense, (46), 235-254 (2013)

Alonso-Almeida, M. D. M., y Llach, J. Socially responsible companies: Are they the best workplace for millennials? A crossnational analysis, doi.org/10.1002/csr.1675, Corporate Social Responsibility and Environmental Management, 26(1), 238247 (2019).

Aly, A. H., Debbab, A., y Proksch, P. Fungal endophytes: Unique plant inhabitants with great promises, doi.org/10.1007/s00253-011-3270-y, Applied Microbiology and Biotechnology, 90(6), 1829-1845 (2011)

ASCUN. El pensamiento Universitario Na21 Responsabilidad social universitaria. Revistas ASCUN, 2, 105. (2011)

Bansah, K. J., Dumakor-Dupey, N. K., Stemn, E., y Galecki, G. Mutualism, commensalism or parasitism? Perspectives on tailings trade between large-scale and artisanal and small-scale gold mining in Ghana, doi.org/10.1016/j.resourpol.2018.03.010, Resources Policy, 57, 246-254 (2018)

Barrera-Rodríguez, A. M., y Rodríguez, O. Informal institutions that are a result of governance structure: Internal identification in public, private, and hybrid organizations. Revista ESPACIOS, 39(34) (2018).

Bascompte, J. Mutualism and biodiversity, doi.org/10.1016/j.cub.2019.03.062, Current Biology, 29(11), R467-R470 (2019)

Bueno, E., Salmador, M. P., Rodríguez, Ó., y Martín-De Castro, G. Internal logic of intellectual capital: A biological approach, doi.org/10.1108/14691930610681474, Journal of Intellectual Capital, 7(3), 394-405 (2006)

Caja-Vivancos, S., y García-Gómez, M. J. ¿Parasitismo o simbiosis en las relaciones entre universidad y calle? Issuc: una propuesta práctica de cómo hacer carrera académica colaborando en la transformación del entorno y en la visibilización de todos los agentes sociales. In U. de La Rioja (Ed.), Respuestas transdisciplinares en una sociedad global. Aportaciones desde el Trabajo Social, pp. 1-25. La Rioja, España. (2016)

Castro-Ríos, G. A., y Noguera Hidalgo, Á. L. Depredación organizacional: las consecuencias del uso del poder en la organización, doi.org/10.1016/j.estger.2014.03.001, Estudios Gerenciales, 30(132), 233-242 (2014) 
Chiva-Bartoll, Ò., Santos-Pastor, M. L., y Salvador-García, C. Valoración del impacto del aprendizaje servicio universitario en el ámbito de la actividad física y el deporte: una propuesta desde la teoría de stakeholders, doi.org/10.30827/publicaciones.v49i4.11727, Publicaciones, 49(4), 29-48 (2019).

Clarke, J., Holt, R., y Blundel, R. Re-imagining the growth process: (co)-evolving metaphorical representations of entrepreneurial growth, doi.org/10.1080/08985626.2014.888099, Entrepreneurship and Regional Development, 26(3-4), 234-256 (2014)

Clarkson, M. E. A stakeholder framework for analyzing and evaluating corporate social performance, doi.org/10.5465/amr.1995.9503271994, Academy of Management Review, 20(1), 92-117 (1995)

Coccia, M. A theory of classification and evolution of technologies within a Generalised Darwinism, doi.org/10.1080/09537325.2018.1523385, Technology Analysis and Strategic Management, 31(5), 517-531 (2019).

Cutovoi, I. T. M. Creating sustainable value (csv) and innovation process under stakeholder theory, doi.org/10.14807/ijmp.v9i3.405, Independent Journal of Management \& Production, 9(3), 1044 (2018).

Dos Santos, L. F., dos Santos Nobre, A. C., Resende, T. C., y Martins-Ramos, A. S. (2019). Analysis of stakeholders in the Management of Social Projects, doi.org/10.5585/gep.v10i1.10957, Revista de Gestão e Projetos, 10(1), 37-50 (2019).

Duque-Oliva, E. J. La gestión de la universidad como elemento básico del sistema universitario: una reflexión desde la perspectiva de los stakeholders. Revista Innovar, (Especial Educación), 25-41(2009)

Freeman, R. E. Strategic management: a stakeholder approach (C. U. Press, Ed.). Nueva York, Estados Unidos (2010)

Freeman, R. E., Wicks, A. C., y Parmar, B. Stakeholder theory and "the corporate objective revisited." Organization Science, 15(3), 364-369 (2004).

Ganhao, M. T. Las metáforas organizacionales, doi.org/10.5565/rev/papers.1864, Papers. Revista de Sociologia, 51, 183 (2016)

Garde-Sánchez, R., Rodríguez-Bolívar, M. P., y López-Hernández, A. M. Online disclosure of university social responsibility: A comparative study of public and private US universities, doi.org/10.1080/13504622.2012.749976, Environmental Education Research, 19(6), 709-746 (2013)

Hernández, C. N. J., y Domínguez, Ó. F. C. Perspectivas y consideraciones sobre el aporte biológico al desarrollo de la gestión de tecnología. Innovar, 18(32), 47-64 (2008)

Hilje, L. Simbiosis: consideraciones terminológicas y evolutivas. Uniciencia, 1(1), 57-60 (1984)

Huerta-Riveros, P. C., Gaete-Feres, H. G., y Pedraja-Rejas, L. M. Dirección estratégica, sistema de información y calidad. El caso de una universidad estatal chilena, doi.org/10.4067/s0718-07642020000200253, Información Tecnológica, 31(2), 253-266 (2020).

Lakoff, G., y Johnson, M. Metáforas de la vida cotidiana (2nda edici; Cátedra, Ed.). Madrid, España (1990)

Mainardes, E., Alves, H., y Raposo, M. Identifying stakeholders in a portuguese university: a case study, doi.org/10.4438/1988-592X-RE-2012-362-167, Revista de Educacion, (362), 429-457 (2013)

Mars, M. M., Bronstein, J. L., y Lusch, R. F. The value of a metaphor: Organizations and ecosystems. doi.org/10.1016/j.orgdyn.2012.08.002. Organizational Dynamics, 41(4), 271-280 (2012)

Martínez-Fernández, A. R., y Cordero del Campillo, M. El parasitismo y otras asociaciones biológicas. Parásitos y hospedadores. In M.-H. I. de España (Ed.), doi.org/10.1016/j.micpath.2017.01.013, Parasitologia Veterinaria, 1a edición, pp. 22-38, (2005)

Martínez, M., Serrano, A., Montoya, A., y Montoya, I. La metáfora biológica como metodología de aprendizaje organizacional. El caso de los ecosistemas de innovación. Espacios, 38(43), 18 (2017)

Marulanda, N., y Rojas, M. D. Ethics in higher education institutions to build trusting relationships with stakeholders, doi.org/10.4067/S0718-07642019000300269, Información Tecnológica, 30(3), 269-276 (2019).

Mesly, O. Techno-predation - a contemporary phenomenon jeopardizing innovation networks all over the world, doi.org/10.1080/08276331.2018.1459018, Journal of Small Business and Entrepreneurship, 31(6), $483-493$ (2019).

Misas, G. La educación superior en Colombia. Análisis y estrategias para su desarrollo (1era edici; U. N. de Colombia, Ed.). Bogotá, Colombia (2004)

Mitchell, R. K., Wood, D. J., y Agle, B. Toward a theory of stakeholder identification and salience: defining the principle of who and what really counts, doi.org/10.5465/AMR.1997.9711022105, Academy of Management Review, 22(4), 853-886 (1997)

Mollis, M. Un breve diagnóstico de las universidades argentinas: identidades alteradas. In Clacso (Ed.), Las universidades en América Latina: ¿reformadas o alteradas?. La cosmética del poder financiero, pp. 203-216, Buenos Aires, Argentina (2003)

Montoya-Restrepo, L. A., Montoya-Restrepo, I. A., y Castellanos-Domínguez, O. F. La metáfora organizacional: alternativa de entendimiento procedente de otras ciencias. Revista Facultad de Ciencias Económicas: Investigación y Reflexión, XVIII(1), 75-86 (2010) 
Montoya-Restrepo, L. A., y Montoya-Restrepo, I. A. Aplicación de la metáfora biológica para el desarrollo de formas organizativas en la integración empresarial, doi.org/10.18359/rfce.2163, Revista Facultad de Ciencias Económicas, 20(2), 43-54 (2016)

Osorio, V., y Rodríguez, M. del P. Modelo para la Evaluación de Programas de Ética Organizacional en Universidades, doi.org/10.4067/s0718-07642018000100059, Información Tecnológica, 29(1), 59-70 (2018).

Plichta, J. The co-management and stakeholders theory as a useful approach to manage the problem of overtourism in historical cities - illustrated with an example of Krakow, doi.org/10.1108/IJTC-12-2018-0107, International Journal of Tourism Cities, 5(4), 685-699 (2019).

Robayo, P., Montoya, A., y Castellanos, O. Aportes desde las ciencias biológicas a la teoría de la gestión. Revista Facultad de Ciencias Económicas, XIX(1), 175-192 (2011)

Robinson, R. N. S., Baum, T., Golubovskaya, M., Solnet, D. J., y Callan, V. Applying endosymbiosis theory: Tourism and its young workers, doi.org/10.1016/j.annals.2019.102751, Annals of Tourism Research, 78(June), 102751 (2019).

Saikkonen, K., Faeth, S. H., Helander, M., y Sullivan, T. J. Fungal endophytes: A continuum of interactions with host plants, doi.org/10.1146/annurev.ecolsys.29.1.319, Annual Review of Ecology and Systematics, 29, 319-343 (1998)

Saunders, M., Lewis, P., y Thornhill, A. Research methods for business students (Séptima ed; P. E. Limited, Ed.) (2016).

Siebold, N. Reference points for business model innovation in social purpose organizations: A stakeholder perspective, doi.org/10.1016/j.jbusres.2020.01.032, Journal of Business Research, (January), 1-10, (2020).

Taylor, J. E., Moldoveanu, M., y Taylor, J. L. Product characteristics and the effectiveness of dow's countermeasure for predatory pricing, doi.org/10.1080/13571516.2012.750043, International Journal of the Economics of Business, 20(1), 114 (2013).

Tejani, G. G., Savsani, V. J., y Patel, V. K. Adaptive symbiotic organisms search (SOS) algorithm for structural design optimization, doi.org/10.1016/j.jcde.2016.02.003, Journal of Computational Design and Engineering, 3(3), 226-249 (2016)

Tohidian, I., y Rahimian, H. Bringing Morgan's metaphors in organization contexts: An essay review, doi.org/10.1080/23311975.2019.1587808, Cogent Business and Management, 6(1), 4-11 (2019).

Vallaeys, F., De la Cruz, C., y Sasia, P. M. Responsabilidad social universitaria: manual de primeros pasos (McGRAWHIL). México, DF (2009)

Vieira-Salazar, J. A. La responsabilité sociale des universités publiques colombiennes. Le cas de la fonction de recherche de l'université publique de Manizales. Université de Rouen (2013)

Villaneda, A. Metáfora biológica del proceso creativo en la enseñanza-aprendizaje para la conformación plástica. Iconofacto, 11(16), 22-39 (2015).

Vujnovic, M., y Kruckeberg, D. Imperative for an arab model of public relations as a framework for diplomatic, corporate and nongovernmental organization relationships, doi.org/10.1016/j.pubrev.2005.05.018, Public Relations Review, 31(3), 338-343. (2005)

Zhang, W., Shi, Y., Yang, M., Gu, X., Tang, R., y Pan, X. Ecosystem evolution mechanism of manufacturing service system driven by service providers, doi.org/10.1080/00207543.2017.1305131, International Journal of Production Research, 55(12), 3542-3558 (2017). 
